Parallel Processing Letters Vol. 4 No. 4 (1993) 489-492

(C) World Scientific Publishing Company

\title{
AUTHOR INDEX \\ VOLUME 4 (1994)
}

Ali, H.H. and El-Rewini, H., On the intractability of task allocation in distributed systems

Alnuweiri, H.M., A fast reconfigurable network for graph connectivity and transitive closure

Anderson, R.J. and Simons, B.B., A fast heuristic for loop parallelization

Ayguadé, E., see Valero, M.

Balasa, F., Franssen, F.H.M., Catthoor, F.V.M. and de Man, H.J., Transformation of nested loops with modulo indexing to affine recurrences

Barlos, F. and Frieder, O., Join workload partitioning under uniform and skewed input relations

Beame, P., Kutyłowski, M. and Kik, M., Information broadcasting by exclusive-read PRAMS

Bhagavathi, D., Olariu, S., Shen, W. and Wilson, L., A unifying look at semigroup computations on meshes with multiple broadcasting

Bhanukumar, H., see Trahan, J.L.

Bhatt, P.C.P., see Saxena, S.

Carlier, R., see Germain, C.

Catthoor, F.V.M., see Balasa, F.

Chou, R.-S. and Hsu, L.-H., l-edge fault-tolerant designs for meshes

Clausen, J. and Träff, J.L., Do inherently sequential branch-andbound algorithms exist?

Comellas, F., Fiol, M.A. and Gómez, J., On large vertex symmetric 2-reachable digraphs

Cosnard, M., Editorial note

Cosnard, M., Editorial note

Darte, A. and Robert, Y., On the alignment problem

Das, S.K. and Halverson, R.H., Simple deterministic and randomized algorithms for linked list ranking on the EREW PRAM model

de Man, H.J., see Balasa, F.

Delaplace, F., see Germain, C.

Dhall, S.K., see Yang, S.B.

Diks, K., Malinowski, A. and Pelc, A., Reliable token dispersal with random faults

$4(1994) 149-157$
$4(1994) 105-115$
$4(1994) 281-299$
$4(1994) 405-415$
$4(1994) 271-280$
$4(1994) 95-104$
$4(1994) 159-169$

4 (1994) 73-82

4 (1994) $117-124$

4 (1994) 429-436

4 (1994) 367-378

4 (1994) $271-280$

4 (1994) 385-389

4 (1994) 3-13

4 (1994) 379-384

4 (1994) 1-2

4 (1994) 365-366

4 (1994) 259-270

4 (1994) 15-27

4 (1994) $271-280$

4 (1994) $367-378$

4 (1994) $29-36$

4 (1994) $417-427$ 
Duato, J., A theory to increase the effective redundancy in wormhole networks

El-Rewini, H., see Ali, H.H.

Feautrier, P., Toward automatic distribution

Fiol, M.A., see Comellas, F.

Franssen, F.H.M., see Balasa, F.

Frieder, O., see Barlos, F.

Galley, C.N. and Iliopoulos, C.S., A simple parallel algorithm for the single function coarsest partition problem

Ganapathy, K. and Wah, B.W., Optimal synthesis of processor arrays with pipelined arithmetic units

Gasperoni, F. and Schwiegelshohn, U., Generating close to optimum loop schedules on parallel processors

Germain, C., Delaplace, F. and Carlier, R., A static execution model for data parallelism

Gewali, L.P. and Stojmenovic, I., Computing external watchman routes on PRAM, BSR, and interconnection network models of parallel computation

Gómez, J., see Comellas, F.

Griebl, M. and Lengauer, C., On the space-time mapping of WHILE-loop

Gupta, S.K.S., Huang, C.-H., Sadayappan, P. and Johnson, R.W., Implementing fast fourier transforms on distributed-memory multiprocessors using data redistributions

Halverson, R.H., see Das, S.K.

Heckler, C. and Thiele, L., Computing linear data dependencies in nested loop programs

Hsu, L.-H., see Chou, R.-S.

Huang, C.-H., see Gupta, S.K.S.

Huang, S.-T., see Tsai, M.-S.

Iliopoulos, C.S., see Galley, C.N.

Jerraya, A.A., see Rahmouni, M.

Johnson, R.W., see Gupta, S.K.S.

Johnson, T., see Swift, L.K.

Kelly, W. and Pugh, W., Selecting affine mappings based on performance estimation

Kik, M., see Beame, P.

Kutyłowski, M., see Beame, P.

Lakshmivarahan, S., see Yang, S.B.

Lau, F.C.M., see Xu, C.-Z.

Lengauer, C., Quinton, P., Robert, Y. and Thiele, L., Editorial note

Lengauer, C., see Griebl, M.

Levcopoulos, C. and Przytycka, T.M., A work-time trade-off in parallel computation of Huffman trees and concave least weight subsequence problem

Lin, R., Olariu, S., Schwing, J.L. and Zhang, J.Y., Computing on reconfigurable buses - A new computational paradigm

Livadas, P.E., see Swift, L.K.

Malinowski, A., see Diks, $\mathrm{K}$.

Mihajlović, M.D., see Milovanović, E.I.

4 (1994) $125-138$

4 (1994) 149-157

4 (1994) 233-244

4 (1994) 379-384

4 (1994) 271-280

4 (1994) 95-104

$4(1994) 437-445$

4 (1994) 339-350

4 (1994) 391-403

4 (1994) 367-378

4 (1994) 83-93

4 (1994) 379-384

4 (1994) 221-232

4 (1994) 477-488

4 (1994) 15-27

4 (1994) 193-204

4 (1994) 385-389

4 (1994) 477-488

4 (1994) 65-72

4 (1994) 437-445

4 (1994) 351-364

4 (1994) 477-488

4 (1994) 447-453

4 (1994) 205-219

4 (1994) 159-169

4 (1994) 159-169

4 (1994) 29-36

4 (1994) 139-147

4 (1994) 189-191

4 (1994) 221-232

4 (1994) $37-43$

4 (1994) 465-476

4 (1994) 447-453

4 (1994) 417-427

4 (1994) 53-64 
Milovanović, E.I., Mihajlović, M.D., Milovanović, I.Ž. and Stojčev, M.K., Solving tridiagonal linear systems on MIMD computers

Milovanović, I.Ž., see Milovanović, E.I.

Mongenet, C., Data compiling for systems of uniform recurrence equations

Nivat, M., Ramkumar, G.D.S., Rangan, C.P., Saoudi, A. and

Sundaram, R., Efficient parallel shuffle recognition

O'Brien, K., see Rahmouni, M.

O'Donnell, J.T., A correctness proof of parallel scan

Olariu, S., see Bhagavathi, D.

Olariu, S., see Lin, R.

Pan, Y., A more efficient constant time algorithm for computing the Hough transform

Peiron, M., see Valero, M.

Pelc, A., see Diks, K.

Persiano, G., An optimal algorithm for the dining philosophers problem

Prasad, V.C., see Saxena, S.

Przytycka, T.M., see Levcopoulos, C.

Pugh, W., see Kelly, W.

Quinton, P., see Lengauer, C.

Rahmouni, M., O'Brien, K. and Jerraya, A.A., A loop-based scheduling algorithm for hardware description languages

Ramesh, R., $R^{2}$ : A reconfigurable rewrite machine

Ramkumar, G.D.S., see Nivat, M.

Rangan, C.P., see Nivat, M.

Robert, Y., see Darte, A.

Robert, Y., see Lengauer, C.

Sadayappan, P., see Gupta, S.K.S.

Saoudi, A., see Nivat, M.

Saxena, S., Bhatt, P.C.P. and Prasad, V.C., On parallel prefix computation

Schwiegelshohn, U., see Gasperoni, F.

Schwing, J.L., see Lin, R.

Shen, W., see Bhagavathi, D.

Simons, B.B., see Anderson, R.J.

Stojčev, M.K., see Milovanović, E.I.

Stojmenovic, I., see Gewali, L.P.

Sundaram, R., see Nivat, M.

Swift, L.K., Johnson, T. and Livadas, P.E., Parallel creation of linear octrees from quadtree slices

Thiele, L., see Heckler, C.

Thiele, L., see Lengauer, C.

Träff, J.L., see Clausen, J.

Trahan, J.L. and Bhanukumar, H., Parallel random access machines without boolean operations

Tsai, M.-S. and Huang, S.-T., A self-stabilizing algorithm for the shortest paths problem with a fully distributed demon

Valero, M., Ayguadé, E. and Peiron, M., Network synchronization and out-of-order access to vectors

4 (1994) 53-64

4 (1994) 53-64

$4(1994) 245-257$

4 (1994) $455-463$

4 (1994) $351-364$

4 (1994) $329-338$

4 (1994) 73-82

4 (1994) 465-476

4 (1994) 45-52

4 (1994) $405-415$

4 (1994) 417-427

4 (1994) $181-187$

4 (1994) 429-436

4 (1994) 37-43

4 (1994) 205-219

4 (1994) 189-191

4 (1994) 351-364

4 (1994) $171-180$

4 (1994) 455-463

4 (1994) 455-463

4 (1994) 259-270

4 (1994) 189-191

4 (1994) 477-488

4 (1994) 455-463

4 (1994) 429-436

4 (1994) 391-403

4 (1994) 465-476

4 (1994) 73-82

4 (1994) 281-299

4 (1994) 53-64

4 (1994) 83-93

4 (1994) 455-463

4 (1994) 447-453

4 (1994) 193-204

4 (1994) 189-191

4 (1994) 3-13

4 (1994) 117-124

4 (1994) 65-72

4 (1994) 405-415 
van Dongen, V., Compiling distributed loops onto SPMD code Violard, E., A mathematical theory and its environment for parallel programming

Wah, B.W., see Ganapathy, K.

Wilson, L., see Bhagavathi, D.

Xu, C.-Z. and Lau, F.C.M., Optimal parameters for load balancing with the diffusion method in mesh networks

Yang, S.B., Dhall, S.K. and Lakshmivarahan, S., A processor efficient connectivity algorithm on random graphs

Zhang, J.Y., see Lin, R.
4 (1994) 301-312

4 (1994) 313-328

4 (1994) 339-350

4 (1994) $73-82$

4 (1994) 139-147

4 (1994) 29-36

4 (1994) 465-476 